\title{
Der "Regionalpark" als Handlungsfeld der Metropolitan Governance
}

\author{
Eine Realisierungs-Analyse am Beispiel der Ziircher Glattal-Stadt
}

\section{A "Regionalpark" as a field of action for Metropolitan Governance}

\author{
A case study in the Zurich Glattal-Stadt
}

\section{Kurzfassung}

Das Konzept der Regionalparks wird in Deutschland seit den 90er Jahren in mehreren Metropolregionen als Strategie zur Entwicklung der Freiräume umgesetzt. In der Schweiz fällt die aktive Steuerung und Gestaltung der Freiräume in „urbanen Kulturlandschaften“ noch schwer. Die Realisierungschancen und Hürden für einen Regionalpark in der Schweiz werden am Fallbeispiel der Region Glattal im Raum Zürich untersucht. Dabei wird die Schaffung eines Regionalparks als ein Anwendungsfeld der Metropolitan Governance interpretiert, das drei Handlungsstränge umfasst: Die Strategien, Strukturen und Kultur der Governance.

\begin{abstract}
Several Metropolitan Regions in Germany have adopted Regionalpark Concepts as a strategy to develop open spaces. In Switzerland there is a lack of actively governing and shaping open spaces in urbanised landscapes. The chances and obstacles to realizing a Regionalpark in Switzerland have been analysed based on a case study in the Zurich Glattal Region. The study considers the creation of a Regionalpark as a field of action for Metropolitan Governance. In order to perform Metropolitan Governance succesfull three strands of activity are proposed to be taken into consideration: strategies, structures and the culture of Governance.
\end{abstract}

\section{Einleitung}

Die „verstädterten Kulturlandschaften“ in europäischen Metropolregionen, die sich um die Kernstädte ausbreiten, sind längst kein neuartiges Phänomen mehr. Sie sind, ob wachsend, stagnierend oder schrumpfend, die zentralen Lebens- und Wirtschaftsräume unserer Zeit. Um angesichts dieser Realität eine nachhaltige Raumentwicklung und gestalterische Qualitäten zu erreichen, werden in Deutschland in einigen Metropolregionen seit den 90er Jahren die Entwicklungs- und Steuerungskonzepte der "Regionalparks“ oder „Landschaftsparks“ eingesetzt. Diese Konzepte setzen Freiräume, sowohl innerhalb als auch außerhalb des Siedlungsgebietes als strukturierende Elemente mit sozialen, wirtschaftlichen, ökologischen und gestalterischen Funktionen und Potenzialen für die Region ein.

In der Schweiz hinkt die Diskussion um verstädterte Kulturlandschaften hinterher. Ein Beispiel und zugleich der empirische Anwendungsfall dieses Beitrags ist die Zürcher "Glattal-Stadt“, die zwischen Flughafen Zürich-Kloten und der Stadt Zürich liegt, und Teil der Europäischen Metropolregion Nordschweiz ist. 


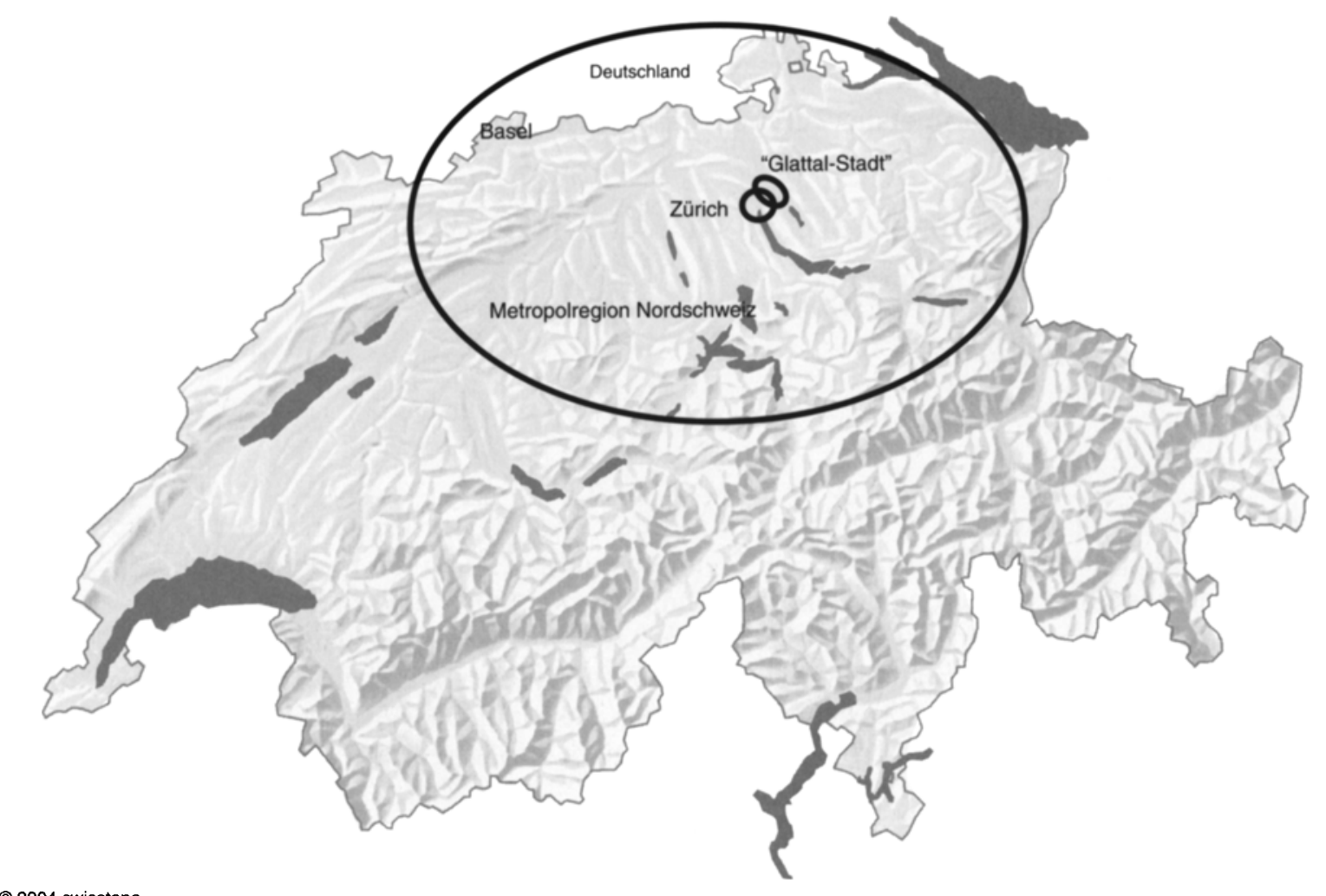

(C) 2004 swisstopo

Wir gehen in dem vorliegenden Beitrag folgender Frage nach: Unter welchen Umständen und mit welchen Hürden lässt sich in der Zürcher Glattal-Stadt innerhalb einer wachstumsstarken, zentralen urbanen Kulturlandschaft ein „Regionalpark-Konzept“ erfolgreich umsetzen?

Der Erkenntnisgewinn aus diesem Artikel besteht darin, anhand der Ergebnisse einer RealisierungsAnalyse die möglichen Ansatzpunkte für einen „Regionalpark Glattal-Stadt“ zu zeigen und aufgrund bestehender Erfahrungen praxisorientierte Lösungen für die Entwicklung vorzuschlagen.

Als konzeptionellen Hintergrund spannen wir die Debatte um die „Metropolitan Governance“ auf. Dieser Diskussion wird das Konzept der Regionalparks an die Seite gestellt und als konkretes Handlungsfeld interpretiert.

Unsere Hypothese lautet, dass die Voraussetzung für die Steuerung der Freiraumentwicklung drei parallele Handlungsstränge eines Governance Modells umfasst, nämlich die Entwicklung von „Strategien“, „Strukturen“ und einer „Kultur“ der Governance. Das Konzept des Regionalparks kann damit als Versuch gelesen werden, raumrelevante Sektoralpolitiken auf ein lokalisierbares Handlungsfeld zu koordinieren. Damit entpuppt sich das Thema als ein lebendiges, aber noch wenig ausgeleuchtetes Anwendungsfeld von Metropolitan Governance.

\section{Die Metropolitan Governance Debatte und die Notwendigkeit eines Handlungsmodells}

\subsection{Die Metropoltian Governance Debatte}

Die Debatte um die "Governance", das heißt die Organisation und Lenkung von Gebietskörperschaften und Institutionen sowie der entsprechenden Verfahren zur Entscheidungsfindung, Mitwirkung und Einflussnahme, wird international geführt (OECD 2001). Die institutionellen Handlungsräume, Zuständigkeitsbereiche von Gebietskörperschaften, funktionalen Zusammenhängen und wirtschaftlichen Verflechtungen überlagern und beeinflussen sich gegenseitig. Die Problemlösungskapazitäten innerhalb dieser so genannten "Multi-Level-Governance“ 
Situation sind häufig mangelhaft, sowohl die vertikale als auch die horizontale Zusammenarbeit weis Lücken auf. Die OECD (2001) hält fest, dass sich eine verbesserte Metropolitan Governance nicht allein durch die Reform von Institutionen und Finanzen ergibt. Vielmehr streicht sie die Veränderung der Haltung und der Entwicklung einer Governance Kultur heraus. Benz und Fürst (2002) gehen davon aus, dass mittels „institutionellen Lernens“ Erfahrungen aus Governance-Projekten schliesslich $\mathrm{zu}$ einer dauerhaften Weiterentwicklung des positiven öffentlichen Rechtes bzw. der Staats- und Verwaltungsstrukturen führen. Healey (2002) führt den Ansatz der „concepts of place" zur Verdeutlichung der räumlichen Dimension regionaler Strategien ein. Mit diesem Ansatz hebt sie die Bedeutung und „gemeinsame Sprache" eines Raumes hervor, die über quantitative Analysedaten hinausgehen. Die Entwicklung von „informal regional initiatives" entlang eines räumlichen Konzeptes ermöglicht den Akteuren aus Politik, Fachplanung und Bevölkerung, Strategien zu entwickeln, die nicht durch sektorale Betrachtungsweisen begrenzt werden. Die "concepts of place" drehen sich dabei um Bedeutung und Identität des Raumes, der als inhaltlicher Aufhänger zur gemeinsamen Erarbeitung von Vorstellungen steht. Damit ergänzen die "concepts of place“ die von der OECD geforderte Governance Kultur und die von Benz und Fürst herausgestrichene Notwendigkeit institutionellen Lernens. Denn die Entwicklung regionaler Zusammenarbeitsformen kann nur dann mobilisierende räumliche Konzepte hervorbringen, wenn die Akteure verschiedener Ebenen sich auf ein gemeinsames Handeln einigen, das die notwendigen Fähigkeiten und Kompetenzen der neuen Organisationsform hervorbringen.

Die Schweiz nimmt sich von der Debatte um mögliche Umstrukturierungsprozesse nicht aus. Sie galt international lange Zeit als Muster der direkten Demokratie, mit ihrem ausgeprägten dezentralen Föderalismus, als Ort vorbildlicher Architektur und als wirtschaftlich erfolgreiches Land. Eisinger und Schneider (2003) konstatieren jedoch, dass dem Denken und Handeln der Bevölkerung und Politikern in der Schweiz zu lange das kriegswirtschaftliche Bewusstsein zugrunde lag, in den Bergen und im ländlichen Raum zu leben. Diese rückwärtsgewandte Haltung hat einen dringenden Reformbedarf hervorgebracht. Gerade in den Metropolregionen ist die Diskussion um die räumliche Struktur des schweizerischen Föderalismus zum wichtigen Thema geworden, da sich hier das Auseinanderklaffen institutioneller Strukturen und räumlicher Problemstellungen deutlich niederschlägt. Die Bun- desregierung hat den städtischen Regionen erst mit der Revision der Bundesverfassung 1997 ihre Aufmerksamkeit geschenkt. Mit der 2001 lancierten Agglomerationspolitik des Bundes begann die finanzielle und technische Förderung von Programmen zur Verbesserung der Situation in den Agglomerationsräumen (Bundesrat 2001).

\subsection{Das Handlungsmodell der Governance}

Damit institutionelles Lernen und mobilisierende räumliche Konzepte sich mit Metropolitan Governance verbinden, schlagen die Autoren ein Handlungsmodell vor, das Elemente des St. Galler Management Konzeptes anwendet (Schwaninger 1997; Rüegg-Stürm 2002; Thierstein et al. 2003). Das Konzept basiert auf einem systemisch-konstruktivistischen Managementansatz. Das Managementkonzept vereinigt die Analyse der Umweltsphären, der Anspruchsgruppen, der Ordnungsmomente, der Entwicklungssituationen, der Interaktion zwischen den Beteiligten am Handlungssystem sowie der Prozessdimension (Rüegg-Stürm 2002; S. 21). Das St.Galler Management Konzept eignet sich für die Governance Debatte besonders gut, weil es die Inhalts- mit der Lernprozessebene der vertikalen und horizontalen Koordination von Sektoralpolitiken integriert. Damit gewinnen die Handlungsträger ein Verständnis über die Steuerungsmöglichkeiten von Entwicklungsprozessen. Es sind die drei folgenden Handlungsstränge, die sich in einem Entwicklungsprozess gegenseitig bedingen müssen:

- Die Strategie beschreibt die Tätigkeiten, welche sich aus dem Selbstverständnis einer Region ergeben, einschliesslich der Funktionen, welche die Region im größeren Ganzen erfüllt. Hier sprechen wir von der Governance-Strategie des Handlungsmodelles.

- Die Strukturen bezeichnen die relativ stabilen Anordnungen in Zeit und Raum. Dabei geht es um Informations- und Managementsysteme im Sinne von Regelwerken, welche die Erfüllung der Aufgaben unterstützen, sowie um Aufbau- und Ablauforganisation. Wir sprechen von der GovernanceStruktur des Handlungsmodelles.

- Die Kultur meint die Verhaltensmuster, insbesondere die ihnen zugrunde liegenden kulturellen Denkweisen, die Werte, Prinzipien und Normen, die wiederkehrenden Routinen und Vertrauensformen. Wir sprechen von der Governance-Kultur des Handlungsmodelles. 


\section{Regionalparks und Landschaftsparks: Mobilisierende Projekte der regionalen Freiraumentwicklung}

\subsection{Die Lesart der "verstädterten Kulturlandschaften“}

Das Anwendungsfeld unseres dreiteiligen Governance Modells ist der Umgang mit Freiräumen in „verstädterten Kulturlandschaften". Es sind jene Regionen, die sich mit ihren ehemals klar abgegrenzten Städten und Dörfern im Umland der Kernstadt zu einem Patchwork aus städtischen und ländlichen Elementen entwickelt haben und sich erheblich vom Mythos der "europäischen Stadt" unterscheiden (Kreibich 2001). Trotz aller Kritik an ihren gestalterischen Qualitäten und ökologischen Defiziten ist es zur Realität geworden, dass die verstädterten Kulturlandschaften im Vergleich zur Kernstadt das grösste Wachstum an Einwohnern und Arbeitsplätzen aufweisen. Auch angesichts einer abklingenden Wachstumsdynamik in den meisten europäischen Staaten, erster Verfallserscheinungen und entstehender Umnutzungs- und Brachflächen ist die "Zwischenstadt” ein Raum, in dem sich eigene Charakteristika und Lebensmuster weiterentwickeln (Sieverts 2003).

Mit dem "Zwischenstadt-Konzept" legte Sieverts (1997; S. 65) diesen Landschafstypen die folgende Sichtweise zugrunde: „...., statt abschätzig von Zersiedlung zu sprechen, kann man eine engmaschige Durchdringung von Freiraum und Siedlung sehen und den Freiraum der Stadtlandschaft als das Verbindende erkennen, mit neuen Gestaltungsperspektiven“.'

\subsection{Der „Regionalpark“ als gestalterisches und strategisches Konzept}

Mit dem Konzept der „Regionalparks“ wurde der bisherigen hoheitlichen Ver- und Gebotsplanung Anfang der 90er Jahre ein aktiver Ansatz der Freiraumentwicklung zur Seite gestellt. Der Begriff "Regionalpark“ ist dabei weder in Deutschland noch in der Schweiz eine planungsrechtlich festgelegte Kategorie. In der Schweiz ruft der Begriff bei vielen Akteuren die Vorstellung einer flächendeckenden, restriktiven Schutzgebietskategorie im ländlichen Raum hervor. Tatsächlich handelt es sich aber um ein ausgesprochen flexibles Konzept, das räumlich und organisatorisch unterschiedlich ausgestaltet wird, einen Mix aus Gestaltung und kreativem Prozess darstellt und sich einer ganzen Reihe planerischer Instrumente bedienen muss. Zu den bekannten Beispielen zählen der „Regionalpark Rhein-Main“ im Frankfurter Raum und der „Emscher Landschaftspark“ im Ruhrgebiet.
Bei allen örtlichen Unterschieden in Bezeichnung, Größe, Design und Charakter ist der Hauptgedanke der Regionalpark-Konzepte die dauerhafte Sicherung, Vernetzung, Gestaltung und Aufwertung der unterschiedlichen Freiräume in der verstädterten Kulturlandschaft über Gemeindegrenzen hinweg. Der gestalterische Leitgedanke der Regionalparks ist, dass es in Zukunft die Landschaftselemente sein werden, die als Netzstruktur dem verstädterten Raum seine Identität geben. Das Freiraumgerüst wird zum „Stadtpark der Region" (Schwarze-Rodrian 2004).

Zur Idee des Regionalparks gehört es, Veränderungen in die gewohnte Umgebung einzufügen und damit eine neue Wirklichkeit für die Wahrnehmung der verstädterten Kulturlandschaft zu schaffen. Die Orte innerhalb und außerhalb der Siedlungsbereiche, die in die Gestaltung einbezogen werden, sind Waldflächen, Landwirtschaftsflächen, Flächen des Naturschutzes, Industriebrachen, städtische Parks und Plätze. Die Nutzungen und Funktionen im Gebiet des Regionalparks werden nicht abgelöst und verdrängt, sondern aufgewertet, koordiniert und übersichtlicher gestaltet: Landwirtschaft, Naturschutz, Langsamverkehr, Gliederung der Siedlungsstruktur, Erholung (Lehmann und Rautenstrauch 2002). Es entstehen gestaltete Wegeverbindungen, besondere Orte, die zum Verweilen einladen, Perlen des Naturschutzes werden als wild gebliebene, naturnahe Orte in Wert gesetzt. Die gestalteten, zugänglichen Freiräume bieten den Bewohnern und Akteuren die Möglichkeit, sich mit ihrer Umgebung zu identifizieren. Dabei bilden die einzelnen Orte, unabhängig davon, ob sie wild und naturnah oder städtisch und „künstlich" geformt sind, Elemente des Regionalparks. Das Motto, das mit dem Regionalpark im Rhein-Main Gebiet geprägt wurde, ist dafür bezeichnend: „Der Landschaft einen Sinn. Den Sinnen eine Landschaft".

Die greifbaren Nutzungs- und Gestaltungsmerkmale, das in der Landschaft sichtbare Produkt des Regionalparks, ist jedoch nur ein Teil des Konzeptes. Hier wird einer der Hauptvorteile und -nutzen des Regionalpark-Konzeptes deutlich: Das im Vergleich zu anderen räumlichen Aufgaben greifbare, sichtbare und attraktive Konzept zur regionalen Gestaltung der Freiräume fördert die fruchtvolle Auseinandersetzung mit den ganz konkreten Qualitäten und Defiziten der verstädterten Kulturlandschaft. Die Entwicklung des Regionalparks mobilisiert und benötigt die drei Handlungsstränge des dreiteiligen Governance Modells: Strukturen zur Organisation der Zuständigkeiten, Strategien zur Entwicklung von Formen und Inhalten der Zusammenarbeit und eine Kultur des gemeinsamen Verständnisses und einer gemeinsamen Vision. 
Hier hilft der Ansatz von Healeys (2002) „concept of place" dabei, den Zusammenhang zwischen und dem Governance Modell und dem Regionalpark-Konzept herzustellen. Danach ist es die Verknüpfung der Aufgaben und Probleme mit der räumlichen Dimension, die gemeinsamen Vorstellungen, eine gemeinsame Sprache und damit die Chance zur Mobilisierung gemeinsamer Handlungen und der Schaffung funktionsfähiger Organisationsformen ermöglichen.

\subsection{Kriterien zur Abgrenzung und Charakterisierung des „Regionalpark“-Begriffs}

Eine Charakterisierung und Abgrenzung des Regionalpark-Begriffes ist gerade vor dem Hintergrund der Diskussion um die aktuelle Teilrevision des Schweizerischen Natur- und Heimatschutzgesetzes notwendig.
Diese sieht die Festschreibung von drei rechtlich festgelegten Parkkategorien, nämlich Landschaftspark, Naturpark und Nationalpark vor (Bundesrat 2002), die sich in vielerlei Hinsicht erheblich von der Idee des Regionalparks unterscheiden. Dieser Vielfalt in der Interpretation der "Park"-Idee trägt die Auswahl der Kriterien Rechnung. Die Abgrenzung des Regionalpark Begriffes wird in der Tabelle anhand der Kriterien Raumbezug, Politischer Wille und Ressourcen, Schutz und Nutzen, Trägerschaft und Recht, Gestaltung und Kommunikation vorgenommen. Die Auswahl der Kriterien folgt den Bedürfnissen nach Abgrenzung der einzelnen Parkkategorien auf Grund der Dimensionen: Raum, Inhalt, Institution und Umsetzung.

Vergleichende Darstellung der drei Parkkategorien des schweizerischen Natur- und Heimatschutzgesetzes und dem Regionalpark-Begriff

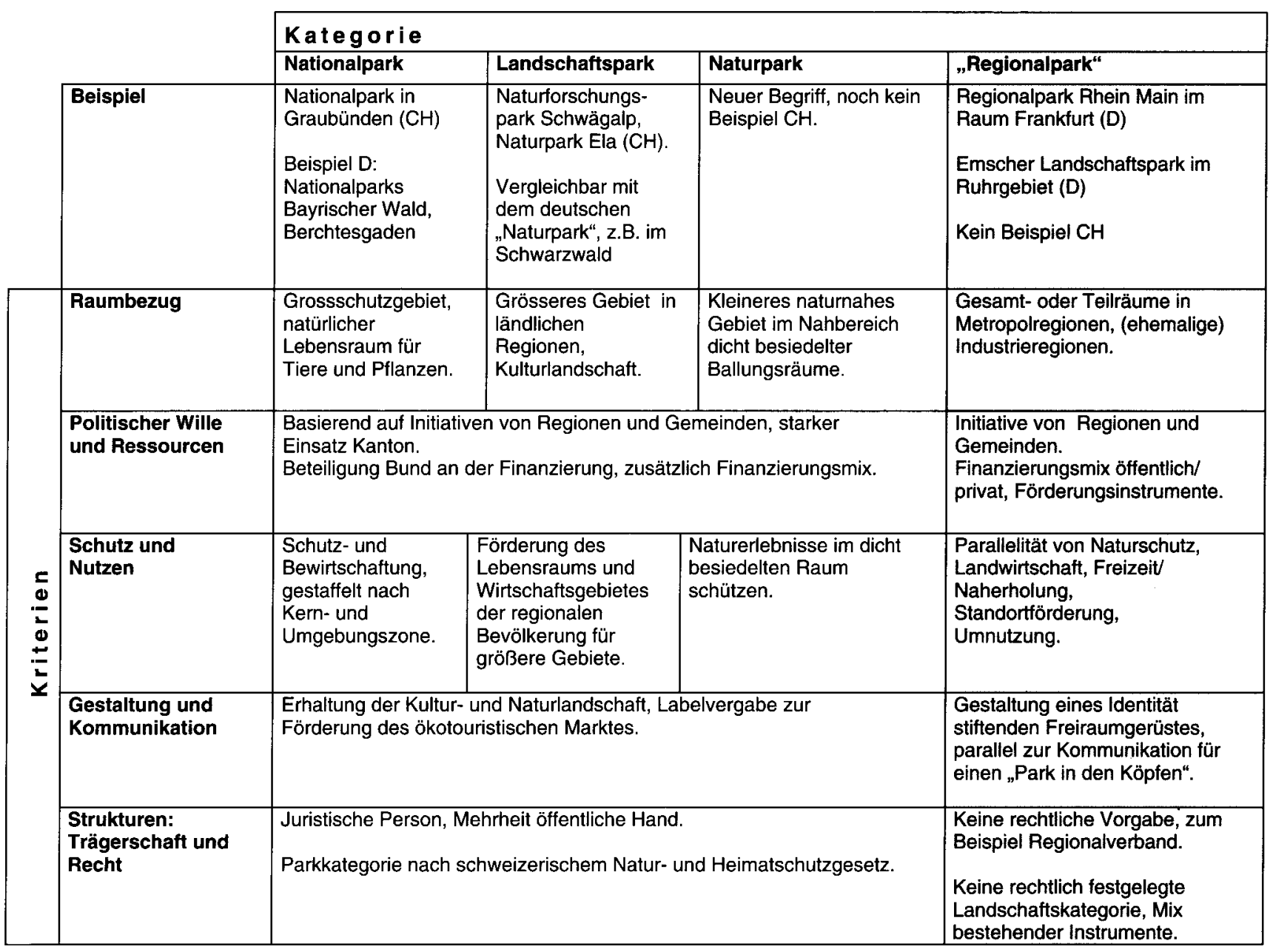

Zusammenstellung: C. Grimaldi und S. Gabi, Grundlagen: Bundesrat 2002a, Lehmann 2003 
Zusammenfassend lassen sich die Unterschiede und Gemeinsamkeiten in der Interpretation und Umsetzung der Park-Idee folgendermassen gegenüberstellen: Zentrales Merkmal der Regionalpark-Idee ist es, die Gesamtheit der verstädterten Kulturlandschaften durch gestaltende und integrierende Eingriffe einzubeziehen. Er wird in Räumen geschaffen, die durch intensiven Nutzungsdruck und Überlagerung von Landwirtschaft, Siedlungsdruck, Verkehr, Naturschutz und Erholung geprägt sind (Lehmann 2003). Zweck eines Regionalpark-Projektes in verstädterten Räumen ist es, einen Integrationsprozess in Gang zu bringen, bei dem die scheinbare Unvereinbarkeit von Natur schützen, wirtschaften und sich erholen widerlegt wird (Bornemann 2001).

Hingegen beziehen sich die schweizerischen Parkkategorien auf den ländlichen Raum oder auf die naturnahen Flächen in der Nähe von Ballungsräumen. Die nationale Bedeutung der Parkgebiete besteht aus „seinem Naturreichtum, aus der besonderen Schönheit und Eigenart seiner Landschaftsteile und aus hochwertigen und einzigartigen Zeugnissen der Kulturlandschaftsentwicklung“ (Bundesrat 2002a: Erläuternder Bericht Teil 2; NHG Neuer Abschnitt 3b, Artikel 23e ).

Die Gemeinsamkeit des Regionalparks und der Parkkategorien besteht darin, dem Grundsatz von Nutzungsinteressen und Schutz und Erhaltung von Natur und Landschaft eine rechtliche Grundlage zu geben. Denn ein Ziel der neuen schweizerischen Parkkategorien ist es, den Schutz der Landschaftsräume in die regionalen Wirtschaftskreisläufe einzubetten und auf dem ökotouristischen Markt anzubieten.

\section{Realisierungs-Analyse: Ein Regionalpark für die Zürcher Glattal-Stadt?}

\subsection{Die Ausgangslage in der Glattal- Stadt}

Der konkrete Anwendungsfall für die Realisierungsanalyse des Regionalpark-Konzeptes ist die Zürcher Glattal-Stadt. Das dicht besiedelte Gebiet zwischen der Stadt Zürich im Süden und dem internationalen Flughafen Zürich im Norden gehört zu den am schnellsten wachsenden Regionen der Schweiz. Auf der politischen Karte liegen hier acht aneinander grenzende Städte und Gemeinden sowie die beiden nördlichen Zürcher Stadtkreise. In der Realität jedoch ist dieser Teil der Agglomeration Zürich, ungeachtet der politischen Grenzen, bereits zu einem zusammenhängenden Siedlungs- und Verkehrsraum geworden. Er ist Wohn- und Arbeitsort für rund 170000 Einwohner und
120000 Beschäftigte (Kanton Zürich 2002), was im europäischen Vergleich gering erscheint, in der Schweiz jedoch die Einwohnerzahl der Städte Basel und Bern übersteigt. Mit dem Entwicklungsmotor des Internationalen Flughafens und der Nähe zum Zentrum der Stadt Zürich ist die Glattal-Stadt Büro-, Kongress-, Einkaufs-, Freizeit-Standort und Verkehrsdrehscheibe für Land- und Luftverkehr. Die Überlagerung von Funktionen geht mit vielfältigen sozialen, ökologischen und wirtschaftlichen Problemen daher, für die ein beträchtliches Handlungsdefizit besteht.

Angesichts des großen Handlungsbedarfs wird im Rahmen der Agglomerationspolitik der Schweizerischen Bundesregierung (Bundesrat 2001) das Modellvorhaben "Netzstadt Glattal“ in der Region Glattal implementiert (Bundesamt für Raumentwicklung 2002). Es wird federführend vom Verein "glow.das Glattal", einem freiwilligen Zusammenschluss aus acht $\mathrm{Ge}$ meinden der Glattal-Stadt sowie Vertretern der kantonalen und Bundesebene getragen.

Das erklärte prozessbezogene Ziel der Akteure des Modellvorhabens ist es, Erfahrungen zur institutionellen Zusammenarbeit zu sammeln und anzuwenden und eine gemeinsame Problemsicht zu entwickeln. Inhaltliches Ziel ist die Gestaltung des Lebensraums "Netzstadt Glattal". Das inhaltliche Ziel haben die Akteure im bisherigen Verlauf des Prozesses durch drei Themenbereiche spezifiziert: „Identifikation mit der Netzstadt Glattal“, „Erholungs- und Naturräume“, "Ortszentren, Wohn- und Arbeitsgebiete in Verbindung mit den künftigen Verkehrsverhältnissen in der Netzstadt Glattal gestalten“.

\subsection{Methodik}

Erkenntnisziel unserer Ex-ante-Realisierungsanalyse war es, die zu erwartenden Schwierigkeiten und Chancen der Umsetzung eines "Regionalparks Glattal-Stadt" innerhalb der urbanen Kulturlandschaft der Metropolregion Zürich zu evaluieren. Die Möglichkeit für diese Analyse entstand aus dem Monitoring des Modellvorhabens "Netzstadt Glattal“. Die Autorin dieses Artikels gehört zu dem externen ProzessmanagementTeam (Team ETH Held), das die Trägerschaft während des zweijährigen Prozesses unterstützt. Erkenntnisse wurden außerdem aus Planungsunterlagen regionaler Institutionen und qualitativen Interviews mit regionalen Akteuren im Rahmen von Untersuchungen zur Glattal-Stadt (Thierstein et al. 2003, Gabi 2003, Team ETH Held 2004) generiert. 
In der Realisierungsanalyse wurde die Hypothese zugrunde gelegt, dass das Regionalpark-Konzept nur dann realisiert werden kann, wenn alle drei miteinander verflochtenen Handlungsstränge des Governance Modells analysiert und die Erkenntnisse in die Implementation einbezogen werden. Das Modellvorhaben eignet sich aufgrund seiner zweifachen Zielsetzung zur Überprüfung dieser Hypothese: Es bezieht sich inhaltlich auf die Gestaltung des Lebensraums, institutionell auf die Governance Thematik.

Die Kriterien aus der Tabelle wurden herangezogen, um die für die Realisierbarkeit des „Regionalparks Glattal-Stadt" relevanten Bereiche zu beleuchten. Im folgenden Kapitel werden die Ergebnisse der Realisierungs-Analyse in verbal-deskriptiver Form entlang dieser fünf Bewertungskriterien zusammengefasst. Daraufhin werden die Folgerungen aus diesen Ergebnissen, bezogen auf die drei Handlungsebenen des Governance Modells, Strategien, Strukturen und Kultur, aufgezeigt.

\subsection{Ergebnisse der Realisierungs-Analyse}

Kriterium „Raumbezug“:

So lokal wie möglich, so regional wie nötig

Die Realität überregionaler Verstädterung übersetzt sich bei den Akteuren nicht von selbst in eine "regionale Identität“ und Problemwahrnehmung. Die Betrachtung der verstädterten Kulturlandschaft der Glattal-Stadt als funktional und phänomenologisch zusammenhängende Siedlungs-, Verkehrs- und Freiraumstruktur widerspricht häufig dem Raumbezug, der politischen Überzeugung und den Handlungen der Träger und Beteiligten des Modellvorhabens.

Bezüglich Freiraumentwicklung bedeutet regionale Koordination für viele die inakzeptable Einmischung von Nachbargemeinden und Kanton in die politischen und planerischen Entscheidungen über die, aus Gemeindesicht optimale, Gewinn bringende Flächennutzung (Gabi 2003). Zugleich gibt es aber auch Beispiele regionaler Kooperation: Im Herbst 2004 beginnen die Verkehrsbetriebe Glattal im Auftrag des Kantons Zürich mit dem Bau einer Stadtbahn, die als roter Faden die Entwicklungsgebiete der Glattal-Stadt erschließen und miteinander verknüpfen wird. Entsprechend des Planungskonzeptes wird sie mit ihrer städtebaulichen Gestaltung auf die umliegenden Gebiete ausstrahlen. Die Initiative für den Bau der Stadtbahn ging von den Glattal-Gemeinden aus, um die Erschließung der zahlreichen neuen Entwicklungsgebiete zu gewährleisten (Planpartner 1991; Thierstein et al. 2003, VBG 2002).
Kriterium „Politischer Wille und Ressourcen“: Die politische Prioritätenliste

Eine Konsequenz aus dem schwachen regionalen Raumbezug ist, dass überkommunale Freiraumentwicklung keine hohe politische Priorität besitzt. Aus Sicht von Politik und Verwaltung in den GlattalGemeinden steht zudem die Lösung und Finanzierung anderer drängender Fragen im Vordergund: das alles überschattende Thema der Fluglärmbelastung und damit einhergehender sinkender Bodenpreise und verminderter Standortqualität, ungelöste Verkehrsprobleme, soziale Probleme durch Kriminalität und mangelnde Integration, drohende Arbeitslosigkeit. Kommunale Politiker arbeiten in der Schweiz zum großen Teil ehren- oder nebenamtlich, häufig parallel in mehreren Gremien und damit unter ständigem Zeitdruck. Angesichts der Vielzahl der anstehenden Probleme bleibt somit wenig Spielraum für neue Aufgaben, die Betroffenen befürchten einen zusätzlichen Arbeits- und Kostenaufwand ohne Mehrwert und Abgeltung. Das Engagement für das Modellvorhaben „Gemeinsame Gestaltung des Lebensraums Netzstadt Glattal“ ist allerdings ein Beleg für den wachsenden politischen Willen zur regionalen $\mathrm{Zu}$ sammenarbeit (Team ETH Held 2004).

Kriterium „Schutz und Nutzen“:

Nutzungskonflikte versus Multifunktionalität

Trotz vielfacher Flächennutzungsansprüche und überlagernder Bedürfnisse in der dicht besiedelten verstädterten Kulturlandschaft der Glattal-Stadt werden Schutz- und Nutzungsfunktionen der Freiräume bisher stark getrennt voneinander betrachtet. Die wahrgenommene Unvereinbarkeit der Nutzungen wird nur wenig in Frage gestellt und verhindert so die offene Abwägung von Interessen. Ein Blick auf die Probleme, welche die Beteiligten des Modellvorhabens „Netzstadt Glattal“ in Bezug auf die Gestaltung des Lebensraums und die Freiraumentwicklung identifiziert haben, ist ein Beleg für die Vielfalt von Freiraumansprüchen und zugleich ihrer bisher ungenügende Koordination: Wenig attraktive Ortszentren, Staus in den Zentren und regionale Verkehrsprobleme, zu wenig Sicherheit und Ordnung, „Ausverkauf“ der Natur, fehlendes Bauland für die Gemeindeentwicklung, Druck auf die Naherholungsflächen, zu wenig Aufenthaltsmöglichkeiten und Freizeitangebote für Jugendliche, Randgruppen und Ausländer, mangelnde Identifikation der Wohn- und Arbeitsbevölkerung mit ihren Gemeinden (Team ETH Held 2004). 
Ansätze für interdisziplinären Umgang mit Freiraumfragen gab es Ende der 90er Jahre, als sich fünf Glattal-Gemeinden zusammenschlossen, um ein gemeinsames, kantonal gefördertes „Landschaftsentwicklungskonzept (LEK)“ ${ }^{\prime} \mathrm{zu}$ erarbeiten. Das Konzept hatte ökologische Vernetzung, aber auch Erholungsaspekte zum Inhalt. Die Umsetzung konzentrierte sich im Weiteren jedoch auf vereinzelte, meist lokale Projekte, die temporäre regionale Projektorganisation wurde nicht weiter geführt (ZPG 2003).

Kriterium „Gestaltung und Kommunikation“: Neues sichtbar und verständlich machen

Gesamthafte Visualisierungen der künftigen Freiraumentwicklung bestehen bisher nicht. Auch im Rahmen des Modellvorhabens arbeiten die Akteure bevorzugt mit "klassischen" zweidimensionalen Plänen, die die Möglichkeiten zur Visualisierung und Entwicklung kreativer, bildhafter Vorstellungen nur wenig einbeziehen. Freiräume werden zwar von einigen Akteuren als identitätsstiftende Elemente der GlattalStadt bezeichnet, Visionen und Darstellungen gibt es nur bezogen auf einzelne Entwicklungsgebiete. Mit der Fokussierung auf die technischen Belange und Notwendigkeiten der Verkehrs- und Siedlungsentwicklung entzieht sich der Vorstellungskraft, dass mit einer veränderten Freiraumgestaltung das alltäglich gewohnte Lebensumfeld auch anders in Erscheinung treten könnte. Der Mehrwert, der durch die Verbindung von Freiräumen und ihre mehrfache Nutzung entsteht, ist für die meisten Akteure noch nicht greifbar und erfahrbar. Auch die Kommunikation von Freiraumbelangen über den Kreis der Umweltfachleute hinaus, etwa an Verantwortliche für Verkehrsund Siedlungsplanung, ist aufgrund der geringen Priorität, aber auch der fehlenden und visionären Überzeugungskraft zurückhaltend (Gabi 2003).

Kriterium „Strukturen: Trägerschaft und Recht“: Zersplitterung der Zuständigkeiten

Ubergreifende Ansätze der Freiraumentwicklung zerfallen in der Glattal-Stadt in die Facetten der autonomen Gemeinden und verschiedenen Zuständigkeitsbereiche von Politik und Verwaltungsressorts. Die Aufgaben des Landschafts- und Naturschutzes liegen bei den kantonalen und kommunalen Naturschutzbehörden und -ämtern. Für fachlich integrierende Ansätze mit Belangen der Siedlungs- und Verkehrsentwicklung und sozialen Aufgaben sind die administrativen und politischen Strukturen wenig ausgeprägt. Die Zürcher Planungsgruppe Glattal (ZPG) ist mit der Aufgabe betraut, räumliche Fragestellungen zwischen Gemeinden und Kanton abzustimmen. Sie besitzt jedoch nur geringe Steuerungswirkung und Ressourcen, zudem gilt regionale Freiraumentwicklung als eher untergeordnetes Thema (Gabi 2003).

\subsection{Folgerungen für die Umsetzung vor dem konzeptionellen Hintergrund des Governance Modells}

Gegenstand der Realisierungs-Analyse waren die Betrachtung der Chancen und Hürden zur Umsetzung eines Regionalpark-Konzeptes. Ausschlaggebend ist, dass der Vorschlag für die Schaffung eines Regionalparks Glattal-Stadt nicht Selbstzweck ist, sondern nach Auffassung der Autoren zahlreiche Vorteile sowohl für die institutionelle als auch die räumliche Situation für diesen Raum beinhaltet: Der Vorteil und Nutzen des Regionalparks liegt auf unterschiedlichen Ebenen. Er beinhaltet gleichzeitig soziale, wirtschaftliche und ökologische Funktionen. Auf der Ebene der Landnutzung lassen sich in verstädterten Kulturlandschaften durch die Schaffung eines Regionalparks landwirtschaftliche Nutzungen, Naturschutz, Erholungsnutzungen, Wegeverbindungen für den Langsamverkehr integrieren. Aus wirtschaftlicher Sicht bringt die Verbesserung der Standortattraktivität Vorteile als Faktor der Wirtschaftsförderung. Als ästhetische und identitätsstiftende Aspekte beinhaltet ein Regionalpark die Wiederherstellung räumlicher $\mathrm{Zu}$ sammenhänge und die Schaffung gestalteter Landschaftselemente in den häufig unzugänglich und als unästhetisch empfundenen Kulturlandschaften. Damit entstehen Orte für Aufenthalt, Kontemplation und sozialen Austausch.

Healey (2002) liefert mit ihrem in Kapitel 2.1 eingeführten "concept of place“ den theoretischen Hintergrund zur Verknüpfung von Governance Fragen mit dem räumlichen Konzept des Regionalparks. Die Schaffung eines regionalen Freiraumsystems ist ein Ausschnitt aus der Fülle komplexer räumlicher Aufgaben und Probleme, der begreifbar ist und sich inhaltlich und räumlich vergleichsweise klar eingrenzen lässt. Nach Healeys Ansatz sind es Prozesse entlang räumlicher Fragestellungen, die es ermöglichen, sektorale Politiken in einen themenbezogenen Zusammenhang $\mathrm{zu}$ bringen.

Um einen Regionalpark Glattal-Stadt Realität werden zu lassen, muss er von den Akteuren nicht nur als technische und gestalterische, sondern als GovernanceAufgabe eingeschätzt werden. Damit würde das Regionalpark Konzept zu einem „,concept of place“, durch dessen mobilisierende Kraft die Akteure sich zur Schaffung von regionalen Organisationsformen und intersektorieller Zusammenarbeit einfinden. 


\section{Strategien: Neues an Bestehendem anknüpfen}

Die Ergebnisse der Realisierungs-Analyse legen die Folgerung nahe, die Aktivitäten der Freiraumentwicklung an bestehende Handlungsfelder und Erfolgsprojekten anzuknüpfen. Wenn auch hinsichtlich regionaler und Sektor übergreifender Zusammenarbeit Defizite bestehen, hat das Beispiel der Stadtbahn Glattal gezeigt, dass eine regionale Sichtweise und Handlungsfähigkeit dann Wirklichkeit wird, wenn der Nutzen für die Gemeinden auf lokaler Ebene einsichtig und greifbar wird. Die Idee der Aufwertung von Freiflächen und vor allem ihrer Erreichbarkeit und Verbindung, kann hier als Pendant zur Stadtbahn als regional verbindendes "grünes Netz" für den Langsamverkehr gesehen werden.

Während der letzten Dekaden ist in der Glattal-Stadt die attraktive Gestaltung der Außenbereiche neuer Bauvorhaben durchaus üblich geworden ist (Planpartner 2002). Nichtsdestotrotz werden bisher neue Entwicklungsgebiete für Wohn- und Arbeitsplätze als mehr oder weniger attrakive „Inseln“ gestaltet, mit Parks und Grünanlagen, möglichst optimiertem Schutz vor Straßenlärm, aber ohne Verbindung zu den Qualitäten der Umgebung. Ausgehend von Außenräumen, in Verbindung mit den Haltestellen der Stadtbahn, können Wegeverbindungen entstehen, die verschiedenartige Freiflächen, Aufenthaltsorte, öffentliche Einrichtungen und Einkaufsmöglichkeiten miteinander verbinden und in ihren regionalen $\mathrm{Zu}$ sammenhang bringen. Hier drängt sich auch die Verbindung auf, die Errungenschaften des oben genannten „Landschaftsentwicklungskonzeptes“ mit bereits erarbeiteten Konzepten und gesicherten Flächen und ökologischen Vernetzungen einzubinden. Das Landschaftsentwicklungskonzept, das in der Sackgasse einer zu sektoriell und kommunal ausgerichteten Sichtweise stecken geblieben ist, könnte durch die geschickte Kombination der Aktivitäten in Form eines Regionalparks den Startpunkt einer neuen Teststrecke markieren.

Strukturen: Prozessgestaltung und inhaltliche Ziele bestimmen die Organisationsform

Die Form der Trägerschaft eines „Regionalparks Glattal-Stadt" richtet sich nach der Notwendigkeit, das Projekt auf eine politisch durchsetzungsfähige Schiene zu stellen und fachliches Know-how und Kreativität einzubinden. Dies kann in Form eines Vereins, einer Gesellschaft, integriert in eine bestehende Gebietskörperschaft oder zunächst einer veränderbaren Adhoc-Organisation. Ausschlaggebend ist, dass die Trägerschaft in den Händen regional einflussreicher Akteure mit einem politischen Willen zum regionalen
Handeln liegen, die „Eingangspforte“ und „Verteiler“ für die Innovation durch einen "Regionalpark GlattalStadt" innerhalb der bestehenden Strukturen und Abläufe sind. Sowohl Akteure aus der Projektleitung der erwähnten Stadtbahn Glattal als auch der Projektleitung des Modellvorhabens „Netzstadt Glattal“ sind Beispiele für potenzielle Träger. Die Trägerschaft muss die Chance nutzen, neben den „klassischen“ politischen und administrativen Vertretern die öffentlichen Träger der Siedlungsentwicklung sowie private Investoren und Entwicklungsgesellschaften „ins Boot“ zu nehmen. Die Kräfte, die in hohem Maße zur Entwicklung und Veränderung der verstädterten Kulturlandschaft beitragen, würden damit bewusst eingebunden und die Siedlungsentwicklung zu einem Motor für die Freiraumentwicklung gemacht.

Da der Regionalpark kein rechtlich festgelegtes Konzept darstellt, muss die formale Verankerung schrittweise erfolgen. Dafür ist ein Mix verschiedener Instrumente, bestehender Schutzkategorien, Leistungsvereinbarungen und privatrechtlicher Verträge sowie temporärer Nutzungsvereinbarungen erforderlich.

Kultur: Eine Kultur der Kreativität und Kommunikationsfähigkeit schaffen

Regionale Kooperation für einen „Regionalpark Glattal-Stadt" benötigt den gemeinsamen Willen und eine Kultur der Zusammenarbeit. Wo es darum geht, den Mehrwert durch Zusammenarbeit und multifunktionale Nutzungen aufzuzeigen, bieten das gestalterische Potenzial und die visuelle Qualität des „Regionalparks Glattal-Stadt“ die Chance, ein Verbindungsstück zu bilden.

Zur Idee des Regionalparks gehört es, Veränderungen in die gewohnte Umgebung einzufügen, die eine neue Wirklichkeit für unsere Wahrnehmung schaffen, lokale Besonderheiten in ein neues Licht zu rücken und regionale Bezüge herzustellen. Das Spektrum der Gèstaltung innerhalb eines Regionalpark-Konzeptes ist vielseitig, die Eigenarten der verstädterten Kulturlandschaft können spektakulär in Szene gesetzt oder zurückhaltend „freigelegt“ werden. Das Bestehende kann hinterfragt und verändert, das Spannungsfeld zwischen Freiräumen und bebauter Landschaft hervorgehoben und profane "Rest"flächen in spezielle Orte verwandelt werden. Die Arbeit mit gestalterischen Mitteln, fotografische, physische und virtuelle Darstellungsmethoden, die Schaffung gemeinsamer Visionen und ihre bildhafte Darstellung wird bisher von Landschaftsarchitekten und an Hochschulen, nicht aber im Alltag von Planung und Politik eingesetzt. 
In Anbetracht der untersuchten politischen und planerischen Strukturen und Akteurskonstellationen ist die Prozessgestaltung ein ebenso wichtiger Erfolgsfaktor, wie es die gestalterischen Ansätze sind. Die Ausgestaltung des Prozesses muss eine intensive begleitende Öffentlichkeitsarbeit, Raum zur gegenseitigen Verständigung über die Idee und, je nach Prozessphase, ein breites Partizipationsangebot beinhalten. Denn entscheidend ist es, im Verlauf des Prozesses immer wieder den „Park in den Köpfen“ (Selle 2000) entstehen zu lassen.

\section{Fazit}

Zu Beginn haben wir die Hypothese zugrunde gelegt, dass die Realisierung eines „Regionalparks GlattalStadt" entscheidend davon abhängt, ob durch die Entwicklung der drei Handlungsstränge des GovernanceModells, der Strategien, Strukturen und Kultur, eine Anschlussfähigkeit zwischen dem Regionalpark-Konzept und den bestehenden Abläufen und der Ausgangslage hergestellt werden kann.

Folgende Erkenntnisse bleiben zum Schluss:

1 Der multifunktionale Charakter des RegionalparkKonzeptes kann ein Schlüssel zur Mobilisierung einer Entwicklungsstrategie und einer Kooperationskultur sein. Die Prüfung, ob durch die integrative Betrachtung regionaler Probleme ein Mehrwert entsteht, wird in Zukunft anhand der Themen des Modellvorhabens bewiesen werden müssen: Verbindung und Aufwertung der Erholungs- und Naturräume, Schaffung von Identifikationsmöglichkeiten in und um die Wohn- und Arbeitsorte, Herstellung und Aneignung neuer Verkehrsverbindungen für den Langsamverkehr.

2 Das Konzept „Regionalpark Glattal-Stadt", hat Chancen auf die Realisierung, wenn es an einem bestehenden regionalen Erfolgsbeispiel anknüpft und somit deutlich wird, dass für jede einzelne Gemeinde greifbare Vorteile aus dem regionalen Handeln entstehen. Das Projekt der Stadtbahn Glattal als „roter Faden“ ist hierfür ein inhaltlicher Aufhänger.

3 Akteure und Organisationsformen mit einem politischen Willen zum regionalen Handeln sind „Eingangspforte“ und "Verteiler“. So leisten sie Vorschub, damit die institutionelle und inhaltliche Innovation eines "Regionalpark Glattal-Stadt" innerhalb der bestehenden Strukturen und Abläufe überzeugender diffundieren kann.
4 Das gestalterische Potenzial des „Regionalparks Glattal-Stadt" ist das Verbindungsstück, um neue Möglichkeiten der Lebensraumgestaltung vorstellbar, sichtbar und kommunizierbar zu machen.

5 Die Form der Trägerschaft richtet sich nach der Notwendigkeit, die Innovation „Regionalpark Glattal-Stadt" auf eine politisch durchsetzungsfähige Schiene zu stellen und die identifizierten relevanten und regional ausgerichteten Akteure einzubinden.

Für die Region der Glattal-Stadt steht fest, dass dem „mehr vom Gleichen“ - dem Denken in expansiven Planungskategorien - neue Ideen, kreative Konzepte und Prozesse zur Seite gestellt werden müssen, um für die zukünftigen Aufgaben gewappnet zu sein. Auch in den im internationalen Vergleich als wirtschaftlich gesunden und mit hoher Lebensqualität ausgestatteten Stadtregionen der Schweiz nehmen soziale, ökologische und wirtschaftliche Probleme zu. Mit zunehmendem Druck zur Steigerung der Lebensqualität im Angesicht von Fluglärmproblematik und sinkender Akzeptanz für den „Identitäts- und Qualitätsverlust“ der Glattal-Stadt zeichnet sich ab, dass Akteure aus Politik, Verwaltung und Wirtschaft nicht in einer Abwartestellung verharren werden. Ein Regionalpark bietet ihnen eine reelle Chance, um die Anschlussfähigkeit zwischen Erprobtem und Bewährtem einerseits und neuen, kreativen Ansätzen andererseits herzustellen (Thierstein und Gabi 2004).

In der Schweiz ist man sich der Konkurrenz zu Regionen im benachbarten Ausland bewusst. Ebenso sind die räumlichen Strategien, nicht zuletzt die bestehenden Beispiele von Landschafts- und Regionalparks in Deutschland, den Fachpersonen in der Schweiz bekannt. Nach Einschätzung der Autoren des vorliegenden Artikels wird die Adaption von Regionalpark-Konzepten für die verstädterten Regionen der Schweiz früher oder später stattfinden, auch wenn es kurzfristig noch starke Vorbehalte gegenüber der freiwilligen "Einschränkung" der Gemeindeautonomie und ein Erfahrungsdefizit in überkommunaler und intersektoreller Zusammenarbeit gibt. Wahrscheinlich ist, dass sich aufgrund des Föderalismus und der direkten Demokratie Organisationsformen herausbilden werden, die sich stark auf den Willen und die Motivation kommunaler Akteure abstützen werden. 


\section{Anmerkung}

(1)

Die Auseinandersetzung mit den Qualitäten und Gestaltungsmöglichkeiten der verstädterten Kulturlandschaften wird spätestens seit Beginn der 90er Jahre thematisiert. Die Debatte hat sich je nach kulturell-analytischem Kontext anders ausgeformt: in den USA mit den Begriffen „Urban Sprawl“ und „Edge City“ (Garreau 1991), in Deutschland, neben Sieverts "Zwischenstadt" (1997) als großräumig wuchernde Stadtlandschaft (Blotevogel 1998, Fürst 1994) oder im französischen Sprachraum als "métropolisation" und „rurbanisation" (Bassand 1997).

\section{Literatur}

Benz, A,; Fürst, D. (2002): Policy learning in regional networks. In: European Urban and Regional Studies 9, S. 21-35

Bornemann, H. (2001): Naturschutz im Ballungsraum. In: Faust, A.; Flagge, I. und Rautenberg, T. (Hrsg.): Der Regionalpark RheinMain. - Darmstadt, S. 24-25

Bundesrat (2002): Bundesgesetz über den Natur- und Heimatschutz, Teilrevision, Entwurf für die Vernehmlassung. - Bern

Bundesrat (2002a): Erläuternder Bericht zur Teilrevision des Natur- und Heimatschutzgesetzes. - Bern

Bundesrat (2001): Agglomerationspolitik des Bundes. - Bern

Bundesamt für Raumentwicklung (ARE) (2002): Agglomerationspolitik: Modellvorhaben: URL: http:/www.are.admin.ch/are/ $\mathrm{de} / \mathrm{raum} /$ politiquedesagglomerations_5/index.html

Eisinger, A.; Schneider, M. (Hrsg.) (2003): Stadtland Schweiz. Untersuchungen und Fallstudien zur räumlichen Entwicklung in der Schweiz. - Basel, S. 8-19

Gabi, S. (2003): Ein Freiflächennetz für die Zürcher Glattal-Stadt. Diplomarbeit NDS Raumplanung, ET'H. - Zürich

Healey, P. (2002): Spatial Planning as a Mediator for Regional Governance: Conceptions of place in the formation of regional governance capacity. In: Knieling, Jörg und Dietrich Fürst (Hrsg.): Regional Governance. Studies in spatial development 2, S. $13-25$

Kanton Zürich (2002): Statistisches Jahrbuch des Kantons Zürich 2002

Kreibich, V. (2001): Die „Europäische Stadt“ aus der Sicht des Raumplaners. In: Rietdorf, W. (Hrsg.): Auslaufmodell Europäische Stadt? - Berlin, S. 41-55

Lehmann, M. (2003): Strategischer Planungsansatz Freiraumentwicklung in deutschen Stadtregionen, Europäische Hochschulschriften. - Frankfurt (M)

Lehmann, M.; Rautenstrauch, L. (2002): Regionalpark und Regionalmanagement - Synergien zwischen zwei neueren regionalplanerischen Handlungsansätzen. In: DISP (3) 150, S. 46-53

OECD (2001): Cities for Citizens. Improving Metropolitan Governance. - Paris
OECD (2002): Territorial Reviews. Switzerland. Territorial Development Policy Committee. - Paris

Planpartner (1991): Glattal wohin? Entwicklungsplanung Glattal. Bericht 2: Massnahmen. - Zürich

Planpartner (2002): Informationsplan Glattal-Stadt. Bericht. - Zürich

Rüegg-Stürm, J. (2002): Das neue St. Galler ManagementModell. - Bern

Schwaninger, M. (1997): Reflexion über Veränderungsprozesse aus der Managementperspektive. In: Thierstein, A.; Walker, D.; Behrendt, H.; Egger, U.K. Tatort Region. - Baden-Baden, S. $83-104$

Schwarze-Rodrian, M. (2004): Das Neue Emschertal als Leitprojekt. In: Garten und Landschaft 7, S. 12-15

Selle, K. (2000): Vom sparsamen Umgang zur nachhaltigen Entwicklung. - Dortmund

Sieverts, T, (1997): Zwischenstadt. Zwischen Ort und Welt, Raum und Zeit, Stadt und Land. Bauwelt Fundamente 118. - Braunschweig, Wiesbaden

Sieverts, T. (2003): Mitten am Rand. In: Polis 3 (15), S. 36-38

Team ETH Held (2004): The Best Practice Model „Networkcity Glattal " in the Zurich Metropolitan Region, Switzerland. A Workin-process report from Governance practice in Switzerland. Congress-Paper for the International Society for city and regional planning (IsoCaRP), CD-ROM

Thierstein, A.; Held, T.; Gabi, S. (2003): City of Regions: Improving Territorial governance in the Zurich "Glatttal-Stadt“. In: Koll-Schretzenmayr, M.; Keiner, M.; Nussbaumer, G. (Hrsg.) (2003): The Real and Virtual Worlds of Spatial Planning. - Berlin, Heidelberg, New York, S. 111-125

Thierstein, A.; Gabi, S. (2004): When Creativity meets Metropolitan Governance. In: DISP (3) 150, S. 34-40

Verkehrsbetriebe Glattal (2002): Das Ziel ist ein leistungsfähiges Gesamtverkehrssystem, Stadtbahngazette

Zürcher Planungsgruppe Glattal (ZPG)(2003): Gutachten Landschaft im Glattal, Planpartner AG. - Zürich

Dipl.-Geogr. Simone Gabi

Raumplanerin ETH

ETH Hönggerberg

CH-8093 Zürich

Prof. Dr. oec. Alain Thierstein

TU München

Lehrstuhl für Raumentwicklung

Gabelsbergerstr. 30

80290 München 